\title{
Wann muss der Herrscher schweigen? Zur Behandlung königlicher Machtsprüche in der Preußischen Rechtsreform
}

\begin{abstract}
The present paper shall provide an analysis of the discourse regarding Machtsprüche ('dictums') in the elaboration of the codification of the Prussian private law - the "Allgemeines Landrecht für die Preußischen Staaten" - towards the end of the 18 $8^{\text {th }}$ century. A Machtspruch consisted of an authoritative decision by the monarch through which he could intervene in on-going judicial proceedings in civil law matters either by giving a ruling himself or by instructing the court to come to a certain decision. In Prussia the question arose of whether Machtsprüche should be forbidden by law. The paper will reflect the positions of King Frederick II., King Frederick William II., high-ranking Prussian civil servants, and legal reformists in this discourse. It will show that the discourse concerning this question is in a way a continuation of the dichotomy between power on the one hand and law on the other, that was typical of Enlightened Absolutism.
\end{abstract}

\section{Einleitung}

Fast scheint es, als habe es im Preußen des 18. Jahrhunderts für Machtsprüche - also königliche Eingriffe in die Zivilrechtsprechung - eine eindeutige Regelung gegeben. Im Jahre 1752 notierte Friedrich der Große nämlich in seinem Politischen Testament:

„Ich habe mich entschlossen, niemals in den Ablauf der Gerichtsverfahren einzugreifen: in den Gerichten sollen die Gesetze sprechen und der Herrscher hat zu schweigen. ${ }^{\prime 1}$

Mag dies zunächst nach einem unbedingten Verzicht auf Machtsprüche klingen, so ist im Hinblick auf eine solche Sichtweise jedoch eine gewisse Zurückhaltung geboten. Bereits die Tatsache, dass der Preußenkönig hier verlautbaren ließ, sich entschlossen zu haben, deutet eine gewisse Freiwilligkeit an, die durchaus Raum für Ausnahmefälle ließ. Und tatsächlich war

\footnotetext{
${ }^{1}$ Friedrich II., Politisches Testament von 1752, 257.
}

Friedrichs Regierungszeit keineswegs frei von königlichen Interventionen in Gerichtsverfahren. Vor diesem Hintergrund mag es kaum verwundern, dass die Regelung des Verhältnisses zwischen Rechtsprechung und königlicher Macht in Preußen zu einem echten Politikum werden musste, als es Ende des 18. Jahrhunderts um die Frage ging, ob und auf welche Weise die zu entwerfende Kodifikation - das „Allgemeine Landrecht für die Preußischen Staaten" von 1794 - Machtsprüche regeln sollte. Mit der vorliegenden Untersuchung soll dieser Diskurs nachvollzogen und die These belegt werden, dass die ursprünglich in der Reform geplante Machtspruchregelung keine inhaltliche Neuerung gebracht hätte, aber gleichwohl aus der Sicht bestimmter politischer Kräfte zu streichen war.

\section{Die Preußische Rechtsreform}

Bereits zu Beginn des 18. Jahrhunderts hatte es in Preußen Ansätze gegeben, eine als untragbar 
empfundene Rechtszersplitterung durch eine umfassende Rechtsreform zu beenden. $\mathrm{Zu}$ einer nachhaltigen Bemühung um diese Pläne kam es jedoch erst in den letzten Regierungsjahren Friedrichs des Großen, als dieser den schlesischen Justizminister Johann Heinrich Casimir von Carmer zum preußischen Großkanzler ernannte. ${ }^{2}$ Mit Carmer betrat ein Protagonist die politische Bühne der preußischen Staaten, der aktiv für eine umfassende Reform des Rechtswesens eintrat. ${ }^{3}$ Am 14. April 1780 erging die entsprechende königliche Kabinettsorder, die Carmer mit der Durchführung einer solchen Reform betraute. ${ }^{4}$ Der neue Großkanzler und sein Arbeitsstab - unter ihnen als engster Mitarbeiter Carl Gottlieb Svarez - erhielten die Aufgabe, das bisher geltende Recht zu sammeln, am Maßstab des Naturrechts zu messen und den preußischen Verhältnissen anzupassen. ${ }^{5}$

In Anbetracht der immensen Aufgabe, mit der Carmer und sein Arbeitsstab betraut wurden, mag es nicht verwundern, dass es immerhin bis ins Jahr 1788 dauern sollte, bis der erste umfassende Entwurf der preußischen Kodifikation veröffentlicht wurde. ${ }^{6}$ Auf Grundlage einer Beteiligung der Öffentlichkeit wurde dieser Entwurf überarbeitet und mit Patent vom 20. März 1791 als „Allgemeines Gesetzbuch für die PreuBischen Staaten ${ }^{\prime 7}$ der Öffentlichkeit vorgestellt. Es entsprach der grundsätzlichen Intention seiner Verfasser, alle Verhältnisse, welche vor preußischen Gerichten Gegenstand eines Strafverfahrens oder eines Rechtsstreits zwischen Untertanen werden konnten, rechtlich zu regeln. ${ }^{8}$ Das Allgemeine Gesetzbuch von 1791 umfasste dementsprechend unter anderem

\footnotetext{
2 BARZEN, Entstehung 19.

${ }^{3}$ Vgl. KuHLI, Svarez 126.

${ }^{4}$ Friedrich II., Kabinettsorder vom 14. April 1780, 37-

41; vgl. HUCKO, 200. Geburtstag $1449 f$.

${ }^{5}$ SCHREIBER, Gesetz 86.

${ }^{6}$ Vgl. Bussi, Svarez 47.

7 Im Folgenden: „Allgemeines Gesetzbuch“.

${ }^{8}$ LANCIZOLLE, Königthum $46 \mathrm{f}$.
}

Handels-, Gesellschafts- und Arbeitsrecht, Erbrecht, Sachenrecht, Ehe- und Familienrecht, Gemeinde-, Gewerbe- und Baurecht, Beamtenrecht, Lehnsrecht, Kirchenrecht sowie Strafrecht, während das Prozessrecht einer gesonderten Regelung vorbehalten blieb. ${ }^{9}$

Als Zeitpunkt für das Inkrafttreten des Allgemeinen Gesetzbuchs war der 1. Juni 1792 vorgesehen, ${ }^{10}$ allerdings verordnete der neue König Friedrich Wilhelm II., der dem 1786 verstorbenen Friedrich II. auf den Thron gefolgt war, ${ }^{11}$ vor Inkrafttreten der Kodifikation zunächst deren Suspension ${ }^{12}$ und später die Umarbeitung des Entwurfs. ${ }^{13} \mathrm{Zu}$ einem der großen Diskussionspunkte wurde in dieser Phase die Frage, wie in der Kodifikation mit sogenannten königlichen Machtsprüchen zu verfahren sei. Im Einzelnen handelte es sich hierbei um Eingriffe des Monarchen in laufende zivilrechtliche Gerichtsverfahren, die entweder durch eine Entscheidung des Königs in der Sache selbst erfolgten oder durch Anweisung an das jeweilige Gericht vorgenommen wurden, eine bestimmte Entscheidung zu fällen. ${ }^{14}$ Die Bezeichnung „Machtspruch“ war seit Ende des 17. Jahrhunderts üblich, wobei im zeitgenössischen Sprachgebrauch der Begriffsteil "Macht" nicht einen Gewaltakt implizierte, sondern lediglich den Ausspruch der höchsten Machtinstanz bedeutete. ${ }^{15}$ Mit $\S 6$ hatten Carmer und seine Mitarbeiter eine Regelung in die Einleitung des Allgemeinen Gesetzbuchs eingefügt, die nach ihrem Wortlaut Machtsprüche für unverbindlich erklärte. Dort heißt es:

\footnotetext{
${ }^{9}$ KuHLI, Svarez $132 \mathrm{ff}$.

${ }^{10}$ Friedrich Wilhelm II., Publikationspatent vom 20. 3. 1791, VI.

11 Wolf, Große Rechtsdenker 447.

${ }^{12}$ Geheimes Staatsarchiv Preußischer Kulturbesitz (Berlin), I. Hauptabteilung, Repositur 84, Abt. XVI, Nr. 7, Bd. 88, fol. 10r.

${ }^{13}$ Ebd., fol. $45^{\mathrm{r}}-46^{\mathrm{r}}$.

${ }^{14}$ Vgl. SVAREZ, Kronprinzenvorträge 236.

${ }^{15}$ BORNHAK, Rechtsgeschichte 252.
} 
„Machtsprüche, oder solche Verfügungen der obern Gewalt, welche in streitigen Fällen, ohne rechtliches Erkenntniss, ertheilt worden sind, bewirken weder Rechte noch Verbindlichkeiten."

Die Bestimmung bezog sich ausdrücklich nur auf streitige Fälle, also zivilrechtliche Prozesse. ${ }^{16}$ Aus der Formulierung „ohne rechtliches Erkenntniss" ergibt sich, dass $\S 6$ auf solche Machtsprüche beschränkt war, die im laufenden Verfahren ergingen. ${ }^{17}$

Im Zuge der von Friedrich Wilhelm II. angeordneten Umarbeitung - der sogenannten Schlussrevision - rückten einige Bestimmungen der Kodifikation in den Mittelpunkt der Diskussion, unter ihnen auch §6. Carmers Arbeitsstab sah sich schließlich gezwungen, diese Bestimmung zu entfernen, sodass die endgültige Kodifikation von 1794 keinerlei Machtspruchregelung enthielt. Auf die Hintergründe dieser Entwicklung wird im Folgenden einzugehen sein. Was die Kodifikation in ihrer Gesamtheit anbelangt, reicht an dieser Stelle ein Hinweis darauf, dass sie am 1. Juni 1794 unter dem neuen Titel „Allgemeines Landrecht für die Preußischen Staaten" in Kraft trat ${ }^{18}$ - mithin mehr als 14 Jahre, nachdem die Reformarbeiten in Angriff genommen worden waren, und mit ungefähr 19.000 Paragraphen eine der umfangreichsten Kodifikationen der Neuzeit. ${ }^{19}$

\section{Motive und Interessen im Machtspruchdiskurs}

Betrachtet man die eben dargestellte Ereignisgeschichte, so drängt sich der Verdacht auf, dass

\footnotetext{
${ }^{16}$ CONRAD, Rechtsstaatliche Bestrebungen 25.

${ }^{17}$ FinKENAUER, Allgemeines Gesetzbuch 101.

${ }^{18}$ Geheimes Staatsarchiv Preußischer Kulturbesitz (Berlin), I. Hauptabteilung, Repositur 84, Abt. XVI, Nr. 7, Bd. 88, fol. 193r

${ }^{19}$ SCHWENNICKE, Tradition 456.
}

sich Friedrich Wilhelm II. einer öffentlichen Festschreibung eines Machtspruchverbots widersetzt hat, die von seinem Vorgänger gewünscht oder zumindest geduldet worden war. Allerdings erweist ein Blick auf die Motive der beiden Könige und anderer Beteiligter, dass insoweit ein stärker differenzierter Blick auf den Diskurs um die Machtspruchregelung erforderlich ist.

\section{Friedrich II.}

Friedrichs Machtspruchverzichte, die er in seinem Politischen Testament von 1752 - aber auch in demjenigen von $1768^{20}$ sowie gegenüber der Öffentlichkeit ${ }^{21}$ - erklärte, waren zu ihrer Zeit keineswegs selbstverständlich. ${ }^{22}$ So war die königliche Rechtsprechung Mitte des 18. Jahrhunderts durchaus noch üblich, ${ }^{23}$ wenngleich auch nicht mehr unumstritten. ${ }^{24}$ Montesquieu war es gewesen, der mit seinem "Esprit des lois“ von 1748 die Auffassung von der Unzulässigkeit königlicher Machtsprüche vertrat. ${ }^{25}$ Friedrich II. rekurrierte zur Begründung seines Machtspruchverzichts darauf, dass der Herrscher bereits aufgrund seiner beschränkten zeitlichen Kapazitäten nicht in der Lage sei, höchstpersönlich Recht zu sprechen. ${ }^{26}$ Allerdings sah sich der König durch die von ihm erklärten Machtspruchverzichte keineswegs dazu veranlasst, die Justiz bei ihrer Arbeit unkontrolliert zu lassen. ${ }^{27}$ So betonte er etwa die Notwendigkeit, das Verhalten der unteren Gerichtsbehörden regelmäßig durch Kommissare des Obersten Gerichtshofs

\footnotetext{
${ }^{20}$ Friedrich II., Politisches Testament von 1768, 465.

${ }^{21}$ Antwort Friedrichs des Großen vom 2.12. 1774 gegenüber einem Supplikanten, in: KIRCHEISEN, Ansprache 415.

22 HeINRICH, Friedrich II. 256.

${ }^{23}$ NEUfELD, Justizreform $14 \mathrm{f}$.

${ }^{24} \mathrm{Vgl}$. SCHWENNICKE, Entstehung 148f.

${ }^{25}$ BORNHAK, Rechtsgeschichte 252f.; vgl. MONTESQUIEU, Vom Geist der Gesetze 216f.

${ }^{26}$ Friedrich II., Politisches Testament von 1768, 257.

${ }^{27}$ OGRIS, Friedrich der Große 81.
} 
überprüfen zu lassen. ${ }^{28}$ Zudem sollte den einzelnen Prozessparteien weiterhin unbenommen bleiben, sich mit Beschwerden an den König zu wenden. ${ }^{29}$ Bereits hierdurch deutet sich Friedrichs Tendenz an, die durch den Machtspruchverzicht selbst auferlegte Bindung gegebenenfalls $\mathrm{zu}$ überschreiten. ${ }^{30}$ Sollte die Beschwerde einer Prozesspartei nämlich nicht nur Anlass zur Gesetzesänderung für zukünftige analoge Rechtsfälle sein, sondern dem Beschwerdeführer zugleich unmittelbare Abhilfe verschaffen, so implizierte dies die Möglichkeit eines Machtspruchs. ${ }^{31}$ Und tatsächlich lassen sich in Friedrichs Regierungszeit Beispiele finden, in denen er persönlich in Gerichtsverfahren intervenierte. $\mathrm{Zu}$ nennen ist etwa sein Verhalten im sogenannten Müller-Arnold-Prozess im Jahre 1779, in dem Friedrich von der Justiz verlangte, zugunsten des genannten Müllers ein Urteil abzuändern. Da sich die Justiz nicht bereit zeigte, diesem Willen zu entsprechen, ordnete der Monarch unter anderem die Amtsenthebung und Inhaftierung mehrerer hoher Richter an. ${ }^{32}$ Zwar wird in der Forschung zum Teil darauf hingewiesen, der König habe hier letztlich keinen Machtspruch erklärt, sondern lediglich die strafrechtliche Entscheidung gegen die beteiligten Richter verschärft, ${ }^{33}$ doch kann nicht zweifelhaft sein, dass Friedrich in diesem Prozess auf die Zivilrechtsprechung Einfluss zu nehmen suchte. ${ }^{34}$ Von einem Schweigen des Königs im Gericht, wie er es in seinem Politischen Testament von 1752 angekündigt hatte, konnte somit keine Rede sein.

\footnotetext{
${ }^{28}$ Friedrich II., Politisches Testament von 1752, 257; ders., Politisches Testament von 1768, 465.

${ }^{29}$ Friedrich II., Politisches Testament von 1752, 329.

${ }^{30}$ SCHWENNICKE, Entstehung 157.

${ }^{31}$ Vgl. KUHLI, Svarez 207.

32 HattenhaUer, ALR 4.

${ }^{33}$ Vgl. SCHWENNICKE, Entstehung 162.

${ }^{34}$ Vgl. HeInRICH, Friedrich II. 256; KunISCH, Friedrich der Große 298.
}

Friedrich hatte in den Müller-Arnold-Prozess eingegriffen, weil er verhindern wollte, dass dem genannten Müller Unrecht geschieht. ${ }^{35}$ Bindungen, denen sich der Herrscher unterwarf, standen stets unter dem Vorbehalt, dass eine Einhaltung dieser Bindung nicht $\mathrm{zu}$ einem ungerechten Ergebnis führen dürfe. Ein solcher Vorbehalt wird besonders deutlich, wenn man den Kontext betrachtet, in dem der Machtspruchverzicht im Politischen Testament von 1752 erklärt wurde. Dort heißt es nämlich:

„Selbst Recht zu sprechen, ist ein Dienst, mit dem sich kein Herrscher belasten kann [...]. Die zahllosen Einzelheiten eines jeden Falles würden die Zeit aufzehren, die er vornehmlich den anderen Geschäften der Regierung zuwenden muß. [...] Ich habe mich entschlossen, niemals in den Ablauf der Gerichtsverfahren einzugreifen: in den Gerichten sollen die Gesetze sprechen und der Herrscher hat zu schweigen". ${ }^{36}$

Mit dieser Begründung verweist Friedrich darauf, dass die zeitliche Kapazität eines Herrschers nicht ausreicht, um höchstpersönlich Recht zu sprechen. Was der Sache nach durchaus richtig ist, lässt andererseits Raum für Ausnahmen. Denn wenn Friedrich hier auf die Unmöglichkeit höchstpersönlicher Rechtsprechung in der Gesamtheit aller Rechtsstreitigkeiten rekurrierte, so schloss diese Aussage nicht aus, dass es dem Herrscher durchaus möglich war, im Einzelfall in eigener Person Recht zu sprechen. Der Grund, den Friedrich hier für seinen Machtspruchverzicht darlegt, weist damit zugleich auf die faktischen Grenzen der Bindungswirkung hin. ${ }^{37}$

Diese Aspekte dürften auch Friedrichs Ansicht zur öffentlichen Festschreibung einer Macht-

\footnotetext{
${ }^{35}$ Dass die Behauptungen Arnolds - wie sich erst später herausstellen sollte - nicht in allen Punkten der Wahrheit entsprachen, wusste der König offensichtlich nicht (vgl. KuHLI, Svarez 125).

${ }^{36}$ Friedrich II., Politisches Testament von 1752, 257.

37 Vgl. KuHLI, Svarez $205 f$.
} 
spruchregelung in der preußischen Kodifikation beeinflusst haben. Friedrich, der in seiner Selbstwahrnehmung davon ausging, stets richtig zu regieren, bedurfte aus seiner Sicht keinerlei Schranken. Allerdings muss hier auch seine Einstellung gegenüber anderen Herrschern berücksichtigt werden. Mochte er im Hinblick auf seine Person von der Überzeugung durchdrungen gewesen sein, dass er eine Politik aus Staatsräson und zum Wohle der Untertanen verfolgte, so konnte er hinsichtlich anderer Monarchen nicht die gleiche Gewissheit besitzen. Diese Skepsis betraf sowohl zeitgenössische Herrscher anderer europäischer Staaten, an denen Friedrich fast nie mit Kritik sparte, ${ }^{38}$ als auch seinen Nachfolger auf dem preußischen Thron, Friedrich Wilhelm II. Gerade im Hinblick auf dessen Person dürfte die öffentliche Festschreibung von Herrscherpflichten, wie sie Carmers Arbeitsstab beabsichtigte, aus der Perspektive Friedrichs II. durchaus sinnvoll erschienen sein.

Allerdings konnten Verstöße gegen öffentlich festgeschriebene Herrscherpflichten aus Friedrichs Sicht auch in Bezug auf seine Nachfolger nicht sanktioniert werden. Das Herrschaftskonzept des Preußenkönigs sah keinerlei Instanzen vor, die einen Verstoß des Monarchen gegen seine naturrechtlichen Pflichten festzustellen oder zu sanktionieren befugt gewesen wären. Einzig der Urteilsspruch der Nachwelt findet bei ihm an verschiedenen Stellen seiner Werke als sanktionierende Instanz Erwähnung. ${ }^{39}$ Darüber hinaus gab es allerdings für Friedrich keinen irdischen Richter über seine Taten. Insbesondere fehlt es in seinem Herrschaftsverständnis an der Zulassung eines Widerstandsrechts der Untertanen gegen den Monarchen. ${ }^{40}$ Entscheidungsinstanz hinsichtlich der Frage der Einhaltung naturrechtlicher Herrscherpflichten blieb damit

\footnotetext{
${ }^{38}$ Friedrich II., Politisches Testament von 1752, 451.

${ }^{39}$ Friedrich II., Geschichte meiner Zeit 83; DERS., Antimachiavell 486.

${ }^{40} \mathrm{Vgl}$. GoOCH, Frederick the Great 273.
}

allein der König selbst. Vor diesem Hintergrund ist im Folgenden nochmals der Blick auf die Machtspruchregelung nach $\S 6$ der Einleitung des Allgemeinen Gesetzbuchs zu richten.

\section{Die Machtspruchregelung im Allgemeinen Gesetzbuch}

Es spricht einiges dafür, dass die Machtspruchregelung des Allgemeinen Gesetzbuchs im Einklang mit den Ansichten Friedrichs des Großen stand. Wenn es in $\S 6$ heißt, dass Machtsprüche in bestimmten Fällen „weder Rechte noch Verbindlichkeiten" bewirken, richtete sich dies zwar in programmatischer Weise gegen Eingriffe des Herrschers in laufende Rechtsstreitigkeiten. Allerdings kann darin nicht bereits ein sanktionierbares Machtspruchverbot gesehen werden. ${ }^{41} \mathrm{Um}$ dies zu verdeutlichen, sei an dieser Stelle auf einige Reflexionen verwiesen, die Carl Gottlieb Svarez - der Hauptmitarbeiter in Carmers Arbeitsstab - entwickelt hat. In einer Vorlesung, die Svarez dem preußischen Kronprinzen und späteren König Friedrich Wilhelm III. gehalten hat, erklärte jener zum Verhältnis des Herrschers zur Justiz:

„Machtsprüche wirken weder Rechte noch Verbindlichkeiten. Es kann also weder irgendein Minister noch der Souverän selbst Machtsprüche tun." ${ }^{42}$

Zur Begründung dieser Rechtsfolge bezieht sich Carmers Mitarbeiter auf das Erfordernis, die Freiheit der preußischen Untertanen zu wahren. Die unmittelbare Ausübung richterlicher Tätigkeit durch den Regenten sei gefährlich, da man von ihm weder fordern noch erwarten könne, dass ihm alle einzelnen Rechtsnormen seines Landes bekannt seien. Darüber hinaus weist Svarez darauf hin, dass die Einmischung des Landesherrn in Angelegenheiten der Zivilrechtspflege dazu führe, dass er sich nur noch in

\footnotetext{
${ }^{41}$ SCHWENNICKE, Entstehung 137f.

42 SVAREZ, Kronprinzenvorträge 236.
} 
vermindertem Maße anderen Regierungsgeschäften widmen könne. ${ }^{43}$

Klingen diese Ausführungen danach, als bewirke ein Machtspruch keine Rechtsfolge, so trügt dieser Schein jedoch. Im Gegenteil betonte Svarez in Übereinstimmung mit Friedrich II., dass dem jeweiligen Richter oder der jeweiligen Prozesspartei das Recht versagt sein solle, einem königlichen Machtspruch Widerstand zu leisten. ${ }^{44}$ Die unbedingte Gehorsamspflicht leitet Svarez aus dem bürgerlichen Vertrag her, in welchem er die Grundlage des preußischen Staatswesens sieht. Danach komme allein der gesetzgebenden Macht die Befugnis zu, die Frage der Zweckmäßigkeit und Geltung eines Gesetzes zu beurteilen. Der Untertan soll hingegen nicht befugt sein, seine subjektive Überzeugung von der Unverbindlichkeit einer staatlichen Rechtsnorm in einer Verweigerung des Gehorsams auszudrücken. Was er allerdings dürfe, sei die Bildung und öffentliche Kundgabe seiner Überzeugung, solange hieraus keine Gefahr für die innere Ruhe und Ordnung des Staates resultiere. ${ }^{45} \mathrm{Im}$ Falle eines ergangenen Machtspruchs blieb den Gerichten hiernach einzig die Möglichkeit, mittels einer Gegenvorstellung beim Herrscher die Aufhebung des Machtspruchs anzuregen. Kam der Monarch dieser Anregung nicht nach, behielt der Machtspruch Verbindlichkeit. ${ }^{46}$ In diesem Fall war die betroffene Prozesspartei faktisch darauf verwiesen, den Wechsel auf dem preußischen Thron abzuwarten und beim jeweiligen Nachfolger des Königs die Aufhebung des Machtspruchs zu begehren. ${ }^{47}$

\footnotetext{
${ }^{43}$ Ebd. 36, 590.

${ }^{44}$ Geheimes Staatsarchiv Preußischer Kulturbesitz (Berlin), I. Hauptabteilung, Repositur 84, Abt. XVI, Nr. 7, Bd. 9, fol. 168r.

${ }^{45}$ SvAREZ, Kronprinzenvorträge 219, $586 f$.

${ }^{46}$ STÖLZEL, Svarez 309-311.

${ }^{47}$ Svarez, extractus monitorum (Geheimes Staatsarchiv Preußischer Kulturbesitz [Berlin], I. Hauptabteilung, Repositur 84, Abt. XVI, Nr. 7, Bd. 72, fol. 20r).
}

Überträgt man diese Sichtweise auf $\S 6$ der Einleitung des Allgemeinen Gesetzbuchs, so lässt sich Folgendes festhalten: Unmittelbare Adressaten dieser Regelung waren weder der König noch die Gerichte. ${ }^{48}$ Die Zielrichtung dieser Bestimmung scheint eher gewesen zu sein, den Parteien eines Prozesses im Vorhinein von der Absendung einer Supplik an den Herrscher abzuraten. Der Machtspruch war unerwünscht, aber letztlich nicht unerlaubt. ${ }^{49}$ Am ehesten dürfte es das Wesen des $\S 6$ treffen, wenn man diese Bestimmung als Publikation eines vom Herrscher erklärten Machtspruchverzichts ansieht. ${ }^{50}$ So gesehen hält sich die Regelung auch durchaus in der Reichweite dessen, was Friedrich II. bereits in seinen Politischen Testamenten hatte verlautbaren lassen.

Die Einhaltung von $\S 6$ konnte in jedem Fall nur durch den Herrscher selbst festgestellt werden, da das Allgemeine Gesetzbuch keine externe Institution vorsah, die unabhängig vom König $\mathrm{zu}$ einer derartigen Kontrolle befugt gewesen wäre. Solange sich der Monarch zur Frage der Wirksamkeit nicht äußerte, lag jedem von ihm erlassenen Hoheitsakt die Vermutung inne, dass der Herrscher die entsprechende Bestimmung als wirksam verstanden wissen wollte. Noch war der Wille des Königs einziger Geltungsgrund des Rechts; die Befugnis des Herrschers zum Erlass von Gesetzen konnte auch nicht durch eine Kodifikation unterbunden werden. ${ }^{51}$ Juristische Bindungen, denen der Herrscher unterworfen war, vermochten sich im Zeitalter der absoluten - auch der aufgeklärt-absoluten Monarchie nicht zu entwickeln. Sie sollten sich erst im Rahmen der konstitutionellen Monarchien durchsetzen.

Wenn sich die Machtspruchregelung des Allgemeinen Gesetzbuchs entsprechend dem eben

\footnotetext{
${ }^{48} \mathrm{Vgl}$. KRAUSE, Überforderung 189.

${ }^{49}$ FinKenAUER, Allgemeines Gesetzbuch 108.

${ }^{50} \mathrm{Vgl}$. KUHLI, Svarez 221.

${ }^{51}$ Hattenhauer, ALR 17.
} 
Gesagten nahtlos in das überkommene preußische Normsystem einfügte, stellt sich die Frage, warum diese Bestimmung gleichwohl aus dem Allgemeinen Gesetzbuch gestrichen wurde. Dies lenkt den Blick auf die von Friedrich Wilhelm II. angeordnete Schlussrevision.

\section{Friedrich Wilhelm II.}

Vor dem Hintergrund der Feststellung, dass § 6 kein rechtlich durchsetzbares Machtspruchverbot darstellte, mag die unter Friedrich Wilhelm II. vorgenommene Streichung dieser Regelung zunächst befremdlich wirken. Allerdings ließe eine solche Sichtweise die Perspektive der potenziellen preußischen Untertanenschaft unberücksichtigt. Es bestand nämlich bei $\S 6$ durchaus die Möglichkeit, dass diese Regelung über ihren tatsächlichen Anwendungsbereich hinausgehend von dritter Seite allzu extensiv ausgelegt oder gar missinterpretiert wird. Indem diese Regelung nämlich als Konsequenz die Unverbindlichkeit normierte, konnte sie unter Umständen dahingehend missverstanden werden, dass sie die Untertanen bzw. die Justiz im Einzelfall zur Verweigerung des Gehorsams berechtigte, wenn ein Machtspruch erging. ${ }^{52}$ Aus der Perspektive des preußischen Königs musste eine solche Möglichkeit als Gefahr erscheinen.

Begünstigt werden musste die Skepsis des Königs dadurch, dass in der Öffentlichkeit Stimmen laut wurden, die in kritischer Weise die Frage aufwarfen, wie die Machtspruchregelung der preußischen Kodifikation zu verstehen sei. So legte etwa der bayerische Beamte Johann Georg Schlosser - ein Schwager Goethes - den Finger in die Wunde, als er in einer 1789 veröffentlichten Abhandlung fragte, ob die Machtspruchregelung des Allgemeinen Gesetzbuchs den Richter und die einzelne Prozesspartei im Falle eines Verstoßes des Herrschers zum Wi-

\footnotetext{
52 Vgl. KRAUSE, Überforderung 132.
}

derstand berechtige. ${ }^{53}$ Zwar ist diese Frage nach dem oben Gesagten in objektiver Hinsicht zu verneinen, doch konnte in dem Zeitpunkt, als über die Schlussrevision debattiert wurde, nicht ausgeschlossen werden, dass ebenso wie Schlosser auch ein preußischer Richter über den Anwendungsbereich von $\S 6$ im Unklaren sein würde. ${ }^{54}$

$\mathrm{Zu}$ erwähnen ist in diesem Zusammenhang auch eine öffentliche Ansprache des Direktors des Berliner Kammergerichts Friedrich Leopold von Kircheisen aus dem März 1792. In dieser Rede kam Kircheisen unter anderem auf Machtsprüche zu sprechen, die er als Herrschaftsinstrument ablehnte. Seine Ausführungen dürften durchaus geeignet gewesen sein, den Argwohn Friedrich Wilhelms II. im Hinblick auf die neue Kodifikation stärker werden zu lassen. Dies gilt vor allem deshalb, weil sich der Kammergerichtsdirektor in seiner Ablehnung königlicher Machtsprüche nicht nur auf die bereits bei Friedrich dem Großen zu findenden Zweckmäßigkeitserwägungen stützte, wonach ein Monarch die Aufgabe der höchstpersönlichen Rechtsprechung aus tatsächlichen Gründen nicht bewältigen könne. Vielmehr rekurrierte Kircheisen ausdrücklich auch auf das Gewaltenteilungspostulat in Montesquieus Werk "L'Esprit des lois“. .55

Es dürften Beispiele wie die von Schlosser und Kircheisen gewesen sein, die die Besorgnis des Königs verstärkt haben, mit dem Allgemeinen Gesetzbuch eine Kodifikation in Kraft zu setzen, die mit $\S 6$ auf den ersten Anschein ein Widerstandsrecht der preußischen Untertanenschaft gegen königliche Herrschaftsakte normierte. Friedrich Wilhelm II. konnte und wollte eine derartige Unsicherheit in der preußischen

\footnotetext{
53 SCHLOSSER, Briefe 175.

${ }^{54} \mathrm{Vgl}$. KuHLI, Svarez 243.

${ }^{55}$ KirCHEISEN, Ansprache 411f.; vgl. MontesQuieU, Vom Geist der Gesetze 216f.
} 
Rechtslage nicht dulden. ${ }^{56}$ So gesehen spricht vieles dafür, dass der Grund für die Schlussrevision nicht in dem tatsächlichen Inhalt des $\S 6$ lag, sondern vielmehr darin, wie diese Bestimmung unter Umständen von Dritten verstanden werden konnte. ${ }^{57}$

Nach alledem könnte man die Auffassung vertreten, dass die in der Schlussrevision angeordnete Streichung von $\S 6$ keinerlei Konsequenzen besaß. Doch ließe eine solche Sichtweise unberücksichtigt, dass der Kodifikation neben der rechtlichen auch eine starke politische Bedeutung beigemessen werden muss. Wäre $\S 6$ nicht der Streichung zum Opfer gefallen, so hätte die preußische Kodifikation in öffentlich zugänglicher Weise die Selbstdisziplinierung der monarchischen Gewalt dokumentiert. Was Carmer und seinen Mitarbeitern nach alledem blieb, war die Hoffnung, den Herrscher auch jenseits von $\S 6$ auf den Weg des Naturrechts zu führen.

\footnotetext{
${ }^{56} \mathrm{Da}$ eine solche Gefahr nur von Bestimmungen auszugehen schien, die sich an herausragender Stelle im Allgemeinen Gesetzbuch - nämlich in dessen Einleitung - befanden, sah man davon ab, inhaltlich ähnliche Regelungen zu streichen, die in der Kodifikation an weniger exponierter Stelle zu finden waren. So kam es, dass der Machtspruchverzicht nach $\S 6$ der Einleitung des Allgemeinen Gesetzbuchs getilgt wurde, während mit $\S 10$ des 13 . Titels des 2 . Teils eine Bestimmung in der Kodifikation erhalten blieb, die festlegte, dass die Begnadigung eines Straftäters durch den König nicht dazu führen dürfe, dass das Opfer der Straftat seines Anspruchs auf privatrechtliche Entschädigung verlustig geht. Offensichtlich wurde hier die Gefahr einer Gehorsamsverweigerung durch die Justiz gegenüber dem Herrscher als weniger groß eingeschätzt, als es bei $\S 6$ der Einleitung des Allgemeinen Gesetzbuchs der Fall war (vgl. KuHLI, Svarez 245).

${ }^{57}$ Vgl. KRAUSE, Überforderung 132.
}

\section{Korrespondenz:}

Milan Kuhli

Goethe-Universität Frankfurt

Grüneburgplatz 1 (Hauspostfach EXC-8), 60323

Frankfurt am Main, Deutschland

milan.kuhli@normativeorders.net

\section{Literatur:}

Allgemeines Gesetzbuch für die Preussischen Staaten, 2 Bde. (Berlin 1791, ND Frankfurt am Main 1985).

Carola BARZEN, Die Entstehung des „Entwurf(s) eines allgemeinen Gesetzbuchs für die Preußischen Staaten“ von 1780 bis 1788 (Konstanz 1999).

Conrad BORNHAK, Preußische Staats- und Rechtsgeschichte (Berlin 1903).

Emilio BussI, Stato e amministrazione nel pensiero di Carl Gottlieb Svarez precettore di Frederico Guglielmo III di Prussia (Milano 1966).

Hermann CONRAD, Rechtsstaatliche Bestrebungen im Absolutismus Preußens und Österreichs am Ende des 18. Jahrhunderts (= Geisteswissenschaften 95, Köln-Opladen 1961).

Hermann CONRAD, Gerd KleinHeYER (Hgg.), Vorträge über Recht und Staat von Carl Gottlieb Svarez (1746-1798) (= Wissenschaftliche Abhandlungen der Arbeitsgemeinschaft für Forschung des Landes Nordrhein-Westfalen 10, Köln-Opladen 1960).

Richard DiETRICH (Hg.), Die politischen Testamente der Hohenzollern (= Veröffentlichungen aus den Archiven Preußischer Kulturbesitz 20, Köln-Wien 1986).

Thomas FINKENAUER, Vom Allgemeinen Gesetzbuch zum Allgemeinen Landrecht - preußische Gesetzgebung in der Krise, in: ZRG GA 113 (1996) 40216.

Friedrich II., Antimachiavell, in: JOST, Gesammelte Werke 482-522.

Friedrich II., Geschichte meiner Zeit, in: JosT, Gesammelte Werke 82-194.

Friedrich II., Kabinettsorder vom 14. April 1780, in: HATTENHAUER, ALR 37-41.

Friedrich II., Politisches Testament von 1752, in: DIETRICH, Testamente 254-461.

Friedrich II., Politisches Testament von 1768, in: DIETRICH, Testamente 462-697.

Friedrich Wilhelm II., Publikationspatent vom 20. März 1791, in: Allgemeines Gesetzbuch 1, VI. 
George P. Gooch, Frederick the Great. The Ruler, The Writer, The Man (London-New York-Toronto 1947).

Hans Hattenhauer, Allgemeines Landrecht für die Preußischen Staaten von 1794 (Neuwied-KriftelBerlin ${ }^{3}$ 1996).

Gerd HeINRICH, Friedrich II. von Preußen. Leistung und Leben eines großen Königs (Berlin 2009).

Elmar HuCKO, Zum 200. Geburtstag des Allgemeinen Landrechts für die Preußischen Staaten, in: Neue Juristische Wochenschrift 1994, 1449-1453.

I[saak] M[arcus] JosT (Hg.), Gesammelte Werke Friedrichs des Großen in Prosa (Berlin 1837).

Friedrich Leopold KIRCHEISEN, Ansprache vom März 1792 gegenüber dem damaligen Kronprinzen vor dem Kammergericht in Berlin, in: Berlinische Monatsschrift (1792/1) 401-416.

Peter KRAUSE, Die Überforderung des aufgeklärten Absolutismus Preußens durch die Gesetzgebung. $\mathrm{Zu}$ den Hemmnissen auf dem Weg zum Allgemeinen Landrecht, in: Günter BIRTSCH, Dietmar WILlOWEIT (Hgg.), Reformabsolutismus und ständische Gesellschaft. Zweihundert Jahre Preußisches Allgemeines Landrecht (=Forschungen zur brandenburgischen und preußischen Geschichte, NF 3, Berlin 1998) 131-211.

Milan KuHLI, Carl Gottlieb Svarez und das Verhältnis von Herrschaft und Recht im aufgeklärten Absolutismus (= Studien zur europäischen Rechtsgeschichte 272, Frankfurt am Main 2012).

Johannes KunISCH, Friedrich der Große. Der König und seine Zeit (München ${ }^{52005) . ~}$

Carl Wilhelm von LANCIZOLLE, Ueber Königthum und Landstände in Preußen (Berlin 1846).
Charles de Montesquieu, Vom Geist der Gesetze. Auswahl, Übersetzung und Einleitung v. Kurt WEIGAND (Stuttgart 1994).

Hans NeufELD, Die friedericianische Justizreform bis zum Jahre 1780 (Göttingen 1910).

Werner OGRIS, Friedrich der Große und das Recht, in: Oswald HAuser (Hg.), Friedrich der Große in seiner Zeit (= Neue Forschungen zur BrandenburgPreußischen Geschichte 8, Köln-Wien 1987) 47-92.

Johann Georg SCHLOSSER, Briefe über die Gesetzgebung überhaupt, und den Entwurf des preußischen Gesetzbuchs insbesondere (Frankfurt am Main 1789).

Hans-Ludwig SCHREIBER, Gesetz und Richter. Zur geschichtlichen Entwicklung des Satzes nullum crimen, nulla poena sine lege (Frankfurt am Main 1976).

Andreas SCHWENNICKE, Die Entstehung der Einleitung des Preußischen Allgemeinen Landrechts von 1794 (= ius commune 61, Frankfurt am Main 1993).

Andreas SCHWENNICKE, Zwischen Tradition und Fortschritt - Zum zweihundertsten Geburtstag des Preußischen Allgemeinen Landrechts von 1794, in: Juristische Schulung (1994) 456-460.

Adolf STÖLzEL, Carl Gottlieb Svarez. Ein Zeitbild aus der zweiten Hälfte des achtzehnten Jahrhunderts (Berlin 1885).

Carl Gottlieb Svarez, Kronprinzenvorträge, in: CONRAD, KLEINHEYER, Vorträge 1-624.

Erik WOLF, Große Rechtsdenker der deutschen Geistesgeschichte (Tübingen ${ }^{41963) \text {. }}$ 